\title{
Tulgeity of Line Graphs
}

\author{
Akbar Ali.M.M (Corresponding author) \\ Department of Mathematics \\ Sri Shakthi Institute of Engineering and Technology \\ Coimbatore 641 062, Tamil Nadu, India \\ Tel: 91-999-427-9835 E-mail: um_akbar@yahoo.co.in \\ S.Panayappan \\ Department of Mathematics, Government Arts College \\ Coimbatore 641 018, Tamil Nadu, India
}

Tel: 91-944-310-9345 E-mail: panayappan@gmail.com

\begin{abstract}
Tulgeity $\tau(G)$ is the maximum number of disjoint, point induced, non acyclic subgraphs contained in $G$. The formula for the tulgeity of the line graph of complete graph and complete bigraph are derived. Also we present an upperbound for the tulgeity of line graph of any graph and we classify the graph for which the upperbound becomes the formula.
\end{abstract}

Keywords: Tulgeity, Line graph

\section{Introduction}

Point partition number (Gray Chartrand, 1971) of a graph $G$ is the minimum number of subsets into which the point-set of $G$ can be partitioned so that the subgraph induced by each subset has property $P$. Dual to this concept of point partition number of graph is the maximum number of subsets into which the point-set of $G$ can be partitioned such that the subgraph induced by each subset does not have the property $P$. Define the property $P$ such that a graph $G$ has the property $P$ if $G$ contains no subgraph which is homeomorphic from the complete graph $K_{3}$. Now the point partition number and dual point partition number for the property $P$ is refered to as point arboricity and tulgeity of $G$ respectively. Equivalently the tulegity is the maximum number of vertex disjoint subgraphs contained in $G$ so that each subgraph is not acyclic. This number is called the tulgeity of $G$ denoted by $\tau(G)$. Also, $\tau(G)$ can be defined as the maximum number of vertex disjoint cycles in $G$. The formula for tulgeity of a complete bipartite graph is given in (Gray Chartrand., 1968). The problems of Nordhaus-Gaddum type for the dual point partition number are investigated in (Anton, 1990).

All graphs considered in this paper are finite and contains no loops and no multiple edges. Denote by $[x]$ the greatest integer less than or equal to $x$, by $|S|$ the cardinality of the set $S$, by $E(G)$ the edge set of $G$ and by $K_{n}$ the complete graph on $n$ vertices. The other notations and terminology used in this paper can be found in (Frank Harary, 1969).

Line graph of a graph $G$ is defined with the vertex set $E(G)$, in which two vertices are adjacent iff the corresponding edges are adjacent in $G$. Since $\tau(G) \leq\left[\frac{p}{3}\right]$, it is obvious that $\tau(L(G)) \leq\left[\frac{q}{3}\right]$. However for complete graph $K_{p}, \tau\left(K_{p}\right)=\left[\frac{p}{3}\right]$.

\section{Main Theorems}

We now present a formula to find tulgeity of line graph of a complete graph.

Theorem 2.1. The tulgeity of line graph of complete graph $K_{n}, \tau\left(L\left(K_{n}\right)\right)=\left[\frac{n(n-1)}{6}\right]$.

Proof

Let $V\left(K_{n}\right)=\left\{v_{1}, v_{2}, \ldots, v_{n}\right\}$ and let $e_{i j}$ be the edge joining the vertices $v_{i}$ and $v_{j}$. i.e., $E\left(K_{n}\right)=\left\{e_{i j}: 1 \leq i \leq n, i+1 \leq j \leq n\right\}$. Clearly by the definition of line graph, $V\left(L\left(K_{n}\right)\right)=E\left(K_{n}\right)=\left\{e_{i j}: 1 \leq i \leq n, i+1 \leq j \leq n\right\}$ and $\left|V\left(L\left(K_{n}\right)\right)\right|=\frac{n(n-1)}{2}$. The edges incident with the vertex $v_{i}$ in $K_{n}(1 \leq i \leq n)$, form a clique (say $\left.K_{i}^{\prime}\right)$ of order $(n-1)$ in $L\left(K_{n}\right)$. Hence $L\left(K_{n}\right)$ contains $n$ cliques $K_{1}^{\prime}, K_{2}^{\prime}, \ldots, K_{n}^{\prime}$ of order $(n-1)$. 


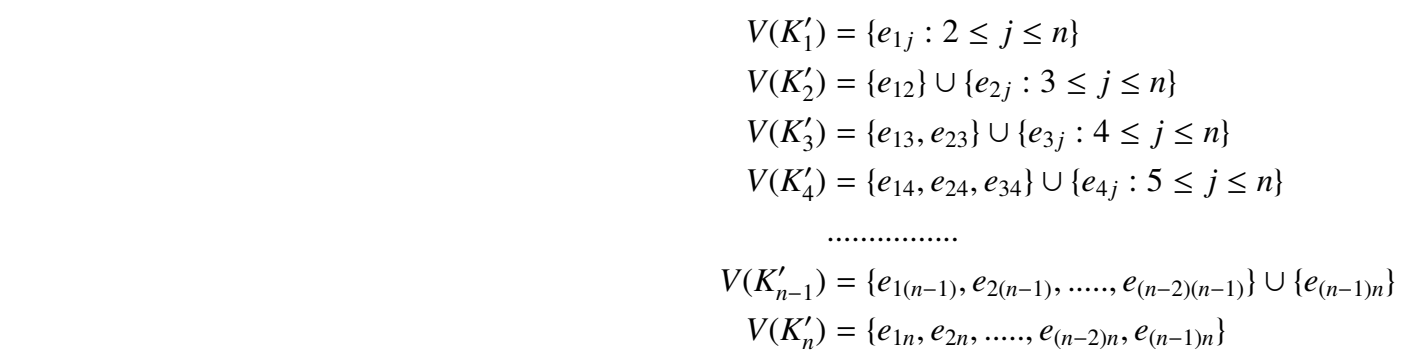

In general $V\left(K_{l}^{\prime}\right)=\left\{e_{i l}: 1 \leq i \leq l-1\right\} \cup\left\{e_{l j}: l+1 \leq j \leq n\right\}$

Case (i) : $n \equiv 1(\bmod 3)$

$(n-1)$ is a multiple of 3 and hence the order of each clique of $L\left(K_{n}\right)$ is a mutiple of 3 . In this case the disjoint cycles of $L\left(K_{n}\right)$ are counted as follows. Consider the following set of cycles of $K_{1}^{\prime}, K_{2}^{\prime}, \ldots, K_{n}^{\prime}$.

Let $C_{1}=\left\{e_{12} e_{13} e_{14}, e_{15} e_{16} e_{17}, \ldots, e_{1(n-2)} e_{1(n-1)} e_{1 n}\right\}$ and let $V\left(C_{1}\right)$ be the set of vertices belonging to the cycles of $C_{1}$. Clearly the cycles of $C_{1}$ are disjoint and $V\left(C_{1}\right)=V\left(K_{1}^{\prime}\right)$. Also $\left|C_{1}\right|=\frac{n-1}{3}$.

Let $C_{2}=\left\{e_{25} e_{26} e_{27}, e_{28} e_{29} e_{210}, \ldots, e_{2(n-2)} e_{2(n-1)} e_{2 n}\right\}$ and let $V\left(C_{2}\right)$ be the set of vertices belonging to the cycles of $C_{2}$. Clearly the cycles of $C_{2}$ are disjoint and $V\left(C_{2}\right)=V\left(K_{2}^{\prime}\right)-\left\{e_{23}, e_{24}, e_{12}\right\}$, where $e_{12} \in V\left(K_{1}^{\prime}\right)$. Also $\left|C_{2}\right|=\frac{n-4}{3}$.

Let $C_{3}=\left\{e_{35} e_{36} e_{37}, e_{38} e_{39} e_{310}, \ldots, e_{3(n-2)} e_{3(n-1)} e_{3 n}\right\}$ and let $V\left(C_{3}\right)$ be the set of vertices belonging to the cycles of $C_{3}$. The cycles of $C_{3}$ are disjoint and $V\left(C_{3}\right)=V\left(K_{3}^{\prime}\right)-\left\{e_{34}, e_{23}, e_{14}\right\}$, where $e_{23} \in V\left(K_{2}^{\prime}\right)$ and $e_{14} \in V\left(K_{1}^{\prime}\right)$. Also $\left|C_{3}\right|=\frac{n-4}{3}$.

Now the vertices $e_{23}, e_{24}$ and $e_{34}$ form a 3 -cycle in $L\left(K_{n}\right)$. Continuing this process we get $\left|C_{4}\right|=\frac{n-4}{3},\left|C_{5}\right|=\frac{n-7}{3}$, $\left|C_{6}\right|=\frac{n-7}{3}, \ldots \ldots,\left|C_{n-5}\right|=1,\left|C_{n-4}\right|=1,\left|C_{n-3}\right|=1,\left|C_{n-2}\right|=0,\left|C_{n-1}\right|=0,\left|C_{n}\right|=0$.

Let $C_{n+1}$ be the set of cycles formed by the vertices of $V\left(K_{i}^{\prime}\right)-V\left(V_{i}^{\prime}\right),(2 \leq i \leq n)$.

i.e., $C_{n+1}=\left\{e_{i(i+1)} e_{i(i+2)} e_{(i+1)(i+2)}: i=3 k-1,1 \leq k \leq \frac{n-1}{3}\right\}$.

Also $\left|C_{n+1}\right|=\frac{n-1}{3}$. Clearly all the cycles of $C_{1}, C_{2}, \ldots, C_{n+1}$ are disjoint and hence the total number of cycles counted by the above process is $\sum_{i}^{n+1}\left|C_{i}\right|=\frac{n(n-1)}{6}$. Hence $\tau\left(L\left(K_{n}\right)\right) \geq \frac{n(n-1)}{6}$.

Case (ii) $: n \equiv 2(\bmod 3)$

$(n-2)$ is a multiple of 3 . In this case the disjoint cycles of $L\left(K_{n}\right)$ are counted as follows. Consider the following set of cycles of $K_{1}^{\prime}, K_{2}^{\prime}, \ldots, K_{n}^{\prime}$. Let $C_{i}$ be the set of cycles of the clique $K_{i}^{\prime}(1 \leq i \leq n)$ and let $V\left(C_{i}\right)$ be the set of vertices belonging to the cycles of $C_{i}$.

Let $C_{1}=\left\{e_{13} e_{14} e_{15}, e_{16} e_{17} e_{18}, \ldots, e_{1(n-2)} e_{1(n-1)} e_{1 n}\right\}$, where $V\left(C_{1}\right)=V\left(K_{1}^{\prime}\right)-\left\{e_{12}\right\}$.

Let $C_{2}=\left\{e_{23} e_{24} e_{25}, e_{26} e_{27} e_{28}, \ldots, e_{2(n-2)} e_{2(n-1)} e_{2 n}\right\}$, where $V\left(C_{2}\right)=V\left(K_{2}^{\prime}\right)$.

Let $C_{3}=\left\{e_{36} e_{37} e_{38}, e_{39} e_{310} e_{311}, \ldots, e_{3(n-2)} e_{3(n-1)} e_{3 n}\right\}$, where $V\left(C_{3}\right)=V\left(K_{3}^{\prime}\right)-\left\{e_{34}, e_{35}\right\}$.

Let $C_{4}=\left\{e_{46} e_{47} e_{48}, e_{49} e_{410} e_{411}, \ldots, e_{4(n-2)} e_{4(n-1)} e_{4 n}\right\}$, where $V\left(C_{4}\right)=V\left(K_{4}^{\prime}\right)-\left\{e_{45}\right\}$.

Clearly the cycles of $C_{1}, C_{2}, C_{3}$ and $C_{4}$ are disjoint and the vertices $e_{34}, e_{35}$ and $e_{45}$ form a 3-cycle. Continuing this process we get $\left|C_{1}\right|=\frac{n-2}{3},\left|C_{2}\right|=\frac{n-2}{3},\left|C_{3}\right|=\frac{n-5}{3},\left|C_{4}\right|=\frac{n-5}{3},\left|C_{5}\right|=\frac{n-5}{3}, \ldots \ldots .,\left|C_{n-5}\right|=1,\left|C_{n-4}\right|=1,\left|C_{n-3}\right|=1 . C_{n-2}$, $C_{n-1}$ and $C_{n}$ are empty. Let $C_{n+1}$ be the set of cycles formed by the vertices of $V\left(K_{i}^{\prime}\right)-V\left(C_{i}^{\prime}\right),(3 \leq i \leq n)$.

i.e., $C_{n+1}=\left\{e_{i(i+1)} e_{i(i+2)} e_{(i+1)(i+2)}: i=3 k, 1 \leq k \leq \frac{n-2}{3}\right\}$.

Clearly $C_{n+1}$ contains disjoint cycles and $\left|C_{n+1}\right|=\frac{n-2}{3}$. Hence minimum number of cycles of $L\left(K_{n}\right)$ is $\sum_{i}^{n+1}\left|C_{i}\right|=$ $\frac{(n+1)(n-2)}{6}$. Since $n \equiv 2(\bmod 3), \frac{(n+1)(n-2)}{6}=\left[\frac{n(n-1)}{6}\right]$. Therefore $\tau\left(L\left(K_{n}\right)\right) \geq\left[\frac{n(n-1)}{6}\right]$. 
Case (iii) $: n \equiv 0(\bmod 3)$

$n$ is a multiple of 3 . In this case the disjoint cycles of $L\left(K_{n}\right)$ are counted as follows.Let $C_{i}$ be the set of cycles of the clique $K_{i}^{\prime}(1 \leq i \leq n)$ and let $V\left(C_{i}\right)$ be the set of vertices belonging to the cycles of $C_{i}$.

Let $C_{1}=\left\{e_{14} e_{15} e_{16}, e_{17} e_{18} e_{19}, \ldots, e_{1(n-2)} e_{1(n-1)} e_{1 n}\right\}$, where $V\left(C_{1}\right)=V\left(K_{1}^{\prime}\right)-\left\{e_{12}, e_{13}\right\}$.

Let $C_{2}=\left\{e_{24} e_{25} e_{26}, e_{27} e_{28} e_{29}, \ldots, e_{2(n-2)} e_{2(n-1)} e_{2 n}\right\}$, where $V\left(C_{2}\right)=V\left(K_{2}^{\prime}\right)-\left\{e_{23}\right\}$. Now $e_{12}, e_{13}$ and $e_{23}$ form a 3-cycle.

Let $C_{3}=\left\{e_{34} e_{35} e_{36}, e_{37} e_{38} e_{39}, \ldots, e_{3(n-2)} e_{3(n-1)} e_{3 n}\right\}$, where $V\left(C_{3}\right)=V\left(K_{3}^{\prime}\right)$.

Let $C_{4}=\left\{e_{47} e_{48} e_{49}, \ldots, e_{4(n-2)} e_{4(n-1)} e_{4 n}\right\}$, where $V\left(C_{4}\right)=V\left(K_{4}^{\prime}\right)-\left\{e_{45}, e_{46}\right\}$.

Let $C_{5}=\left\{e_{57} e_{58} e_{59}, \ldots, e_{5(n-2)} e_{5(n-1)} e_{5 n}\right\}$, where $V\left(C_{5}\right)=V\left(K_{5}^{\prime}\right)-\left\{e_{56}\right\}$.

Now the vertices $e_{45}, e_{46}$ and $e_{56}$ form a 3 -cycle. Continuing this process we get $\left|C_{1}\right|=\frac{n-3}{3},\left|C_{2}\right|=\frac{n-3}{3},\left|C_{3}\right|=\frac{n-3}{3}$ ,....., $\left|C_{n-5}\right|=1,\left|C_{n-4}\right|=1,\left|C_{n-3}\right|=1$. $\left|C_{n-2}\right|=\left|C_{n-1}\right|=\left|C_{n}\right|=0$. Let $C_{n+1}$ be the set of cycles formed by the vertices $V\left(K_{i}^{\prime}\right)-V\left(C_{i}^{\prime}\right),(1 \leq i \leq n)$.

i.e., $C_{n+1}=\left\{e_{i(i+1)} e_{i(i+2)} e_{(i+1)(i+2)}: i=3 k-2,1 \leq k \leq \frac{n}{3}\right\}$ and clearly $\left|C_{n+1}\right|=\frac{n}{3}$. Minimum number of cycles of $L\left(K_{n}\right)$ is $\sum_{i}^{n+1}\left|C_{i}\right|=\frac{n(n-1)}{6}$. Therefore $\tau\left(L\left(K_{n}\right)\right) \geq \frac{n(n-1)}{6}=\left[\frac{n(n-1)}{6}\right]$. Hence in all the three cases $\tau\left(L\left(K_{n}\right)\right) \geq\left[\frac{n(n-1)}{6}\right]$. Now by the very definitions, $L\left(K_{n}\right)$ has $\frac{n(n-1)}{2}$ vertices and hence $\tau\left(L\left(K_{n}\right)\right) \leq \frac{1}{3} \frac{n(n-1)}{2}=\left[\frac{n(n-1)}{6}\right]$. Hence $\tau\left(L\left(K_{n}\right)\right)=\left[\frac{n(n-1)}{6}\right]$.

Each vertex $v_{i}$ of a graph $G$ with degree $\operatorname{deg} v_{i}$ induce a clique of order $\operatorname{deg} v_{i}$ in $L(G)$. i.e., At each vertex $v_{i}$ of $G$, we count $\left[\frac{\operatorname{deg} v_{i}}{3}\right]$ vertex disjoint cycles in $L(G)$. The above argument yields the following result which gives an upperbound for the tulgeity of line graph of any graph.

Theorem 2.2.

$$
\tau(L(G)) \leq \sum_{i}\left[\frac{\operatorname{deg} v_{i}}{3}\right]
$$

The following result characterize the graph for which the above said inequality becomes equal.

Theorem 2.3. If $G$ is a tree and for each pair of vertices $\left(v_{i}, v_{j}\right)$ with deg $v_{i}$, deg $v_{j}>2$, if there exist a vertex $v$ of degree 2 on $P\left(v_{i}, v_{j}\right)$ then $\tau(L(G))=\sum_{i}\left[\frac{\operatorname{deg} v_{i}}{3}\right]$.

Proof. Let $T$ be a graph satisfying the given condition. Each vertex $v_{i}$ of $T$ of degree $>2$ induce a clique of order $d e g v_{i}$ in $L(G)$ and since for each pair of vertices $\left(v_{i}, v_{j}\right)$ of $T$ with degree $>2$, there exists a vertex $v$ of degree 2 on $P\left(v_{i}, v_{j}\right)$, there exists a bridge joining the corresponding cliques in $L(G)$ and hence $\tau(L(G))=\sum_{i}\left[\frac{\operatorname{deg} v_{i}}{3}\right]$.

The tulgeity of complete $n$-partite graphs have been studied by Gary Chartrand et.al in (1968). Here we derive a formula for the tulgeity of line graph of complete bigraph.

Theorem 2.4. The tulgeity of line graph of complete bigraph graph $K_{m, n}, \tau\left(L\left(K_{m, n}\right)\right)=\left[\frac{m n}{3}\right]$.

Proof. Without loss of generality assume that $m \leq n$. Let $V\left(K_{m, n}\right)=\left\{v_{1}, v_{2}, \ldots, v_{m}\right\} \cup\left\{u_{1}, u_{2}, \ldots, u_{n}\right\}$ and $E\left(K_{m, n}\right)=\left\{e_{i j}=\right.$ $\left.v_{i} v_{j}: 1 \leq i \leq m, 1 \leq j \leq n\right\},\left|E\left(K_{m, n}\right)\right|=m n$. By the definition of line graphs, $V\left(L\left(K_{m, n}\right)\right)=\left\{e_{i j}: 1 \leq i \leq m, 1 \leq j \leq n\right\}$ in which $e_{i j}$ and $e_{k l}$ are adjacent if either $i=k$ or $j=l$. Hence $\mid V\left(L\left(K_{m, n}\right) \mid=m n\right.$ and hence $\tau\left(L\left(K_{m, n}\right)\right) \leq\left[\frac{m n}{3}\right]$. Let $V_{i}=\left\{e_{i j}: 1 \leq j \leq n\right\},(1 \leq i \leq m)$. Clearly $\left\langle V_{i}\right\rangle$ is a complete subgraph of $L\left(K_{m, n}\right)$ for each $i$. Similarly if $V_{j}^{\prime}=\left\{e_{i j}: 1 \leq i \leq m\right\},(1 \leq j \leq n)$ then $\left\langle V_{j}^{\prime}\right\rangle$ is a complete subgraph of $L\left(K_{m, n}\right)$.

Case (i):

If $n \equiv 0(\bmod 3)$ then $\left|V_{i}\right| \equiv 0(\bmod 3)$ and hence there exist $\frac{n}{3}$ vertex disjoint cycles in $\left\langle V_{i}\right\rangle$ for each $i,(i \leq i \leq m)$. If $m \equiv 0(\bmod 3)$ then $\left|V_{j}^{\prime}\right| \equiv 0(\bmod 3)$ and hence there exist $\frac{m}{3}$ vertex disjoint cycles in $\left\langle V_{j}^{\prime}\right\rangle$ for each $j,(1 \leq j \leq n)$. In both the cases minimum number of vertex disjoint cycles in $L\left(K_{m, n}\right)$ is $\frac{m n}{3}=\left[\frac{m n}{3}\right]$. Hence $\tau\left(L\left(K_{m, n}\right)\right) \geq\left[\frac{m n}{3}\right]$. 


\section{Case (ii):}

If $n \equiv 1(\bmod 3)$ then $V_{i}-\left\{e_{i n}\right\},(1 \leq i \leq m)$ has $n-1$ vertices for each $i$ and there exist $\frac{n-1}{3}$ vertex disjoint cycles in $V_{i}-\left\{e_{i j}\right\}$ for each $i$. Also, since $\left\langle e_{1 n}, e_{2 n}, . ., e_{m n}\right\rangle$ is a complete subgraph there exist $\left[\frac{m}{3}\right]$ vertex disjoint cycles. Hence $\tau\left(L\left(K_{m, n}\right)\right) \geq \frac{m(n-1)}{3}+\left[\frac{m}{3}\right]=\left[\frac{m n}{3}\right]$.

\section{Case (iii):}

If $n \equiv 2(\bmod 3)$ and $m \equiv 1(\bmod 3)$ then $V_{i}-\left(\left\{e_{i n}\right\} \cup\left\{e_{i(n-1)}\right\}\right),(1 \leq i \leq m)$ has $n-2$ vertices for each $i$ and there exist $\frac{n-2}{3}$ vertex disjoint cycles in $V_{i}-\left(\left\{e_{i n}\right\} \cup\left\{e_{i(n-1)}\right\}\right)$ for each $i$. Also since $m \equiv 1(\bmod 3),\left\langle e_{1(n-1)}, e_{2(n-1)}, . ., e_{m(n-1)}\right\rangle$ has $\frac{m-1}{3}$ vertex disjoint cycles and $\left\langle e_{1 n}, e_{2 n}, \ldots, e_{m n}\right\rangle$ has $\frac{m-1}{3}$ vertex disjoint cycles. Hence $\tau\left(L\left(K_{m, n}\right)\right) \geq \frac{m(n-2)}{3}+\frac{m-1}{3}+$ $\frac{m-1}{3}=\frac{m n-2}{3}=\left[\frac{m n}{3}\right]$.

\section{Case (iv):}

If $n \equiv 2(\bmod 3)$ and $m \equiv 2(\bmod 3)$ then $V_{i}-\left(\left\{e_{i n}\right\} \cup\left\{e_{i(n-1)}\right\} \cup\left\{e_{i(n-2)}\right\}\right),(1 \leq i \leq m)$ has $(n-3)$ vertices for each $i$ and there exist $\frac{n-3}{3}$ vertex disjoint cycles in $V_{i}-\left(\left\{e_{i n}\right\} \cup\left\{e_{i(n-1)}\right\} \cup\left\{e_{i(n-2)}\right\}\right),(1 \leq i \leq m)$ for each $i$. Also $m \equiv 2(\bmod 3)$, $\left\langle e_{1(n-1)}, e_{2(n-1)}, . ., e_{(m-2)(n-1)}\right\rangle$ has $\frac{m-2}{3}$ vertex disjoint cycles and $\left\langle e_{1 n}, e_{2 n}, . ., e_{(m-2) n}\right\rangle$ has $\frac{m-2}{3}$ vertex disjoint cycles. At last the vertices $e_{(m-1)(n-1)}, e_{m(n-1)}, e_{(m-1) n}, e_{m n}$, induces a 4-cycle. Hence $\tau\left(L\left(K_{m, n}\right)\right) \geq \frac{m(n-2)}{3}+2 \frac{m-2}{3}+1=\frac{m n-1}{3}=$ $\left[\frac{m n}{3}\right]$. Hence in all the cases $\tau\left(L\left(K_{m, n}\right)\right) \geq\left[\frac{m n}{3}\right]$. Hence $\tau\left(L\left(K_{m, n}\right)\right)=\left[\frac{m n}{3}\right]$.

\section{References}

Anton Kundrik. (1990). Dual point partition number of complementary graphs. Mathematica Slovaca, 40(4), 367-374.

Frank Harary. (1969). Graph Theory. New Delhi: Narosa Publishing home.

Gray Chartrand., Dennis Geller., \& Stephen Hedetniemi. (1971). Graphs with forbidden subgraphs. Journal of Combinatorial Theory, 10, 12-41.

Gray Chartrand., Hudson V. Kronk., \& Curtiss E. Wall. (1968). The point arboricity of a graph. Israel Journal of Mathematics 6(2), 168-175. 